\title{
»Ändert sich nicht alles um uns herum? Ändern wir uns nicht selbst?« \\ Zum Verhältnis von Leben, Zeit und Gegenwart um 1770
}

I.

Tempora mutantur, et nos mutamur in ipsis - die Zeiten ändern sich und wir uns in ihnen - so lautet ein lateinisches Sprichwort, das seit dem 16. Jahrhundert häufig zitiert wird. Es steht zwar in loser Anlehnung an eine Aussage Ovids in dessen berühmtem naturphilosophischen 15 . Buch seiner Metamorphosen, ${ }^{1}$ wird aber in der Frühen Neuzeit entweder im theologischen Kontext verwendet oder dazu gebraucht, um auf Veränderungen bzw. den Verfall der Sitten zu verweisen, oft in apologetischer Absicht desjenigen, der sich auf das Sprichwort beruft. ${ }^{2}$ Dass die Zeiten sich ändern, ist also ein altes Ar-

1 »Omnia mutantur, nihil interit.« (»Alles verändert sich, nichts geht unter.«) Ovid: Metamorphosen. Lateinisch / Deutsch, übers. und hg. von Michael von Albrecht, Stuttgart 1994, S. 800f.

2 So schreibt Gottfried Schultze in seiner Welt-Beschreibung (1679) im Vorwort an den Leser: "So nun etwa einer oder der ander in andern Chroniken / Historien-Büchern / und Ländern / von den Sitten / anders / alß hierin berühret / selbst gelesen / oder gesehen / der muß dabey das alte Dictum, Tempora mutantur \& nos mutamur in ipsis beobachten / auch zusehen / wie man die Sitten alltäglich endere / denn / es kombt wol / was vor alters gebräuchlich gewesen / sich heute zutage nicht practiciren lässet / dagegen /was bei ihnen übel ausgeschlagen / hie zu zeiten wolgeräht / darumb das treffliche Kunst Stück-

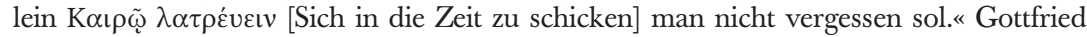
Schultzens neu-augirte Welt-Beschreibung / Darinn Aller Käyserthümer / Königreichen / Republiquen / und der gantzen Welt / Religion / Sitten und Gebräuche; Auch Aller Päbste / Käyser / Könige / und Fürsten auff einander ergangene Succession fein ordentlich und kürtzlich begriffen, Franckfurt a.M. 1679, S. 4. In Lessings Der junge Gelehrte (1747) ist es der Vater, der das Sprichwort zitiert, um seinem Sohn gegenüber zu begründen, warum er vor dem Studium gelehrten Fleiß fordert und danach vor zu viel Gelehrsamkeit warnt: "Chrysander: 'Tempora mutantur mein Sohn - Damis: 'Tempora mutantur? Ich bitte Sie, legen Sie doch die Vorurteile des Pöbels ab. Die Zeiten ändern sich nicht. Denn lassen Sie uns einmal sehen: was ist die Zeit? - - Chrysander: Schweig! Die Zeit ist ein Ding, das ich mir mit deinem unnützen Geplauder nicht will verderben lassen. Meine damaligen Vorschriften waren nach dem damaligen Maße deiner Erfahrung und deines Verstandes eingerichtet." Gotthold Ephraim Lessing: Der junge Gelehrte, in: Ders.: Werke und Briefe in zwölf Bänden, hg. von Wilfried Barner u.a., Bd. 1: Werke 1743-1750, hg. von Jürgen Stenzel, Frankfurt a.M. 1989, S. 139-237, hier S. 146. 
gument und - dafür spricht die Form des Sprichworts - selbst nichts Neues. Wie und mit welcher Tiefendimension, mit welcher Reichweite und in welchem Kontext es allerdings als Argument eingesetzt wird, das ändert sich durchaus erheblich, und zwar in der zweiten Hälfte des 18. Jahrhunderts durch die Bezugnahme auf solche Veränderungen, die das Leben und seine biologischen und seine sozialen Bedingungen betreffen.

In Baron d'Holbachs 1770 erschienenem Buch Das System der Natur ist die Diagnose der permanenten Veränderung auf die raumzeitlichen Bedingungen des physischen Lebens bezogen. Es ändern sich nicht einfach die Sitten und Gebräuche, es ändern sich die grundlegenden Voraussetzungen, die das Leben in den jeweiligen Formen allererst möglich machen. Sowohl die Erde als notwendige Umwelt des Menschen als auch der Mensch, als das Produkt dieser Umwelt, sind in der radikal atheistischen Perspektive Holbachs dem Zufall von Raum und Zeit unterworfen - und nicht Gottes Schöpfungsplan. ${ }^{3}$ Holbach schreibt:

So scheint uns alles zu der Annahme zu berechtigen, daß die menschliche Gattung ein unserm Erdball in der Lage eigentümliches Produkt ist, in der er sich befindet, und daß, wenn diese Lage verändert würde, sich die menschliche Gattung verändern würde oder verschwinden müßte $[\ldots]^{4}$

3 Vgl. hierzu Johannes F. Lehmann: Welt als Umwelt: zur ästhetischen Erfindung eines wissenschaftlichen Konzepts bei Diderot, Goethe und Büchner. Erscheint in: Milieu. Umgebungswissen, hg. von Florian Huber und Christina Wessely. Die ältere, theologisch fundierte Ansicht von der Unveränderbarkeit der Welt formuliert Lessing in seiner als Schüler verfassten Glückwünschungsrede auf 1743: "Niemand leugnet, daß Gott der Schöpfer dieser Welt sei; niemand leugnet, daß Gott die Welt sehr gut erschaffen habe; [...] Hat aber die Welt in ihrer Art die größte Vollkommenheit, so werde ich ohne Bedenken sagen können, daß alles was in der Welt zugleich ist und aufeinander folget, mit einander übereinstimmen müsse; und daß die Welt, so lange sie nach des Schöpfers Willen Welt bleiben soll, keine Hauptveränderung leiden könne." Gotthold Ephraim Lessing: Glückwünschungsrede auf 1743. Bei dem Eintritt des 1743sten Jahres, von der Gleichheit eines Jahres mit dem Andern, in: Ders.: Werke, Bd. 1 (wie Anm. 2), S. 9-18, hier S. 11.

4 Paul Thiry d'Holbach: System der Natur oder von den Gesetzen der physischen und der moralischen Welt. Übers. von Fritz-Georg Voigt, Frankfurt a.M. 1978, S. 78. Das Buch erschien unter falschem Autornamen: Systême de la nature. Première partie. Ou Des loix du monde physique \& du monde moral. Par M. Mirabaud, Londres 1770. Vgl. ebd., S. 84: "Ainsi tout semble nous autoriser à conjecturer que l'espece humaine est une production propre à notre globe, dans la position où il se trouve, \& que cette position venant à changer, l'espece humaine changeroit, ou seroit forcée de disparoître, vû qu'il n'y a que ce qui peut se coordonner avec le tout, ou s'enchaîner avec lui, qui puisse subsister.« 


\section{Und weiter:}

Wenn wir also Veränderungen in der Lage unseres Erdballs annehmen, so unterschied sich vielleicht der ursprüngliche Mensch mehr vom heutigen Menschen als der Vierfüßler vom Insekt. So kann der Mensch ebenso wie alles, was auf unserem Erdball und allen anderen Himmelskörpern existiert, als in einem fortwährenden Wechsel befindlich angesehen werden. So ist uns der Endpunkt der Existenz des Menschen ebenso unbekannt und gleichgültig wie der Anfang. So ist es nicht widersinnig, zu glauben, daß die Gattungen sich unaufhörlich verändern, und wir können unmöglich wissen, was aus ihnen werden wird, noch was sie gewesen sind. ${ }^{5}$

Der Mensch und alle anderen lebenden Organismen sind das Produkt von Lagen (positions), die räumlich und zeitlich variieren und die einen unendlichen Prozess von Veränderungen plausibilisieren, dem auch die Gattungen des Lebens selbst unterworfen sind. In diesem fundamentalen Sinne ist es gemeint, wenn Holbach das alte lateinische Zitat auf Französisch reformuliert: »Ändert sich nicht alles um uns herum? Ändern wir uns nicht selbst? « ${ }^{6}$ Die Blasphemie einer solchen Inanspruchnahme so gewaltiger Zeiträume und die damit verbundene Leugnung der Schöpfung (mit ihrer Gesamtdauer von 6000 Jahren) und der Konstanz der Arten zugunsten der Möglichkeit evolutionärer Entwicklungen ${ }^{7}$ hatte bereits ein Jahr zuvor Diderot in seinem Rêve de d'Alembert begangen. ${ }^{8}$ Während Holbachs Buch nach seinem

5 Holbach: System (wie Anm. 4), S. 79; [Holbach]: Systême (wie Anm. 4), S. 85: »En supposant donc des changemens dans la position de notre globe, l'homme primitif différoit, peut-être, plus de l'homme actuel, que le quadrupede ne differe de l'insecte. Ainsi l'homme, de même que tout ce qui existe sur notre globe \& dans tous les autres, peut être regardé comme dans une vicissitude continuelle. Ainsi le dernier terme de l'existence de l'homme, nous est aussi inconnu \& aussi indifférent que le premier. Ainsi il n'y a nulle contradiction à croire que les especes varient sans cesse, \& il nous est aussi impossible de sçavoir ce qu'elles deviendront, que de sçavoir ce qu'elles ont été."

6 Holbach: System (wie Anm. 4), S. 80; [Holbach]: Systême (wie Anm. 4), S. 86: „Tout ne change-t-il pas autour de nous? Ne changeons-nous pas nous-mêmes? « Entsprechend kommt das korrespondierende Zitat Ovids (Omnia mutantur etc.) mit der naturphilosophischen Theorie des permanenten Wandels der Natur und aller Lebensformen bei Holbach in vollem materialistischen Sinne wieder zu seinem Recht. Vgl. Holbachs Anmerkung 10, ebd., S. 619.

7 Belege zur biblischen Zeitrechnung und Geschichtskonstruktion in der Frühen Neuzeit bei Achim Landwehr: Geburt der Gegenwart. Eine Geschichte der Zeit im 17. Jahrhundert, Frankfurt a.M. 2014, S. 47-89. Siehe auch Helmut Zedelmaier: Der Anfang der Geschichte. Studien zur Ursprungsdebatte im 18. Jahrhundert, Hamburg 2003.

8 Denis Diderot: Le Rêve de d'Alembert, in: Ders.: Euvres complètes, publ. par Herbert Dieckmann, Jacques Proust et Jean Verlot, Paris 1975ff., tome XVII: Le Rêve de d'Alembert. Édition H. Dieckmann et J. Varloot, Paris 1987, S. 115-194. Ich zitiere die deutsche Übersetzung: Denis Diderot: Gespräche mit d'Alembert, in: Ders.: Über die Natur, hg. 
Erscheinen sogleich verboten wurde, verzichtete Diderot auf die Publikation seines Textes, in dem man etwa folgenden Satz daher erst 1830 gedruckt lesen konnte:

Warum bin ich so? Weil ich so werden mußte ... Hier allerdings, aber anderswo? Am Pol, unter dem Äquator, auf dem Saturn? ... Wenn schon eine Entfernung von einigen tausend Meilen meine Art verändert: was wird dann ein Zwischenraum von einigen tausend Erddurchmessern bewirken? ... Und wenn alles ein allgemeiner Strom ist, wie ihn mir das Schauspiel des Universums überall zeigt: was werden dann hier und anderswo die Dauer und die Wandlung einiger Millionen Fahrhunderte nicht alles hervorbringen?

Die Welt als Ort von Möglichkeitsbedingungen zu betrachten, die selbst andauernder Veränderung unterworfen sind, und das Leben bzw. die Lebewesen als das Produkt dieser sich zeitlich verändernden Bedingungen, das wird seit Mitte des 18. Jahrhunderts in verschiedenen Kontexten auch auf die sozialen Bedingungen des Lebens übertragen. Rousseau formuliert es als Begründung für sein Unternehmen, die Prinzipien der Erziehung radikal zu verändern: "Die Verhältnisse ändern sich ständig, der Geist des Jahrhunderts ist unruhig und stürzt von Generation zu Generation alles um. ${ }^{10}$ Es geht hier also nicht um den sprichwörtlichen Verweis auf die Veränderung der Zeiten und Sitten als Rechtfertigung vergangenen Handelns, sondern um ein Argument zur Begründung von Innovationen. Rousseau folgert aus der Tatsache einer sich permanent verändernden sozialen Umwelt, dass der Mensch nicht für einen bestimmten Platz, Stand oder Beruf erzogen werden kann, sondern als ganzer Mensch für alle möglichen Fälle und Rollen, denn, so Rousseau wörtlich, "Leben ist ein Beruf, den ich ihn lehren will. «11 »Vivre est le métier que je lui veux apprendre.«" ${ }^{12}$ Leben impliziert damit die

und mit einem Essay von Jochen Köhler, aus dem Französischen von Theodor Lücke, Frankfurt a.M. 1989, S. 67-143, hier S. 94.

9 Ebd., S. 96f. (Herv. J.L.) Auf S. 97, weiter unten, heißt es: "Ich bin nur deshalb so, weil ich so werden mußte, verändern Sie das Ganze, so verändern Sie notwendig auch mich."

10 Jean-Jacques Rousseau: Emil oder Über die Erziehung, in neuer dt. Fassung besorgt von Ludwig Schmidts, 10. Auflage, Paderborn/Wien/Zürich 1991, S. 15. "Mais, vu la mobilité des choses humaines, vu l'esprit inquiet \& remuant de ce siècle qui bouleverse tout à chaque génération, peut-on concevoir une méthode plus insensée que d'élever un enfant comme n'ayant jamais à sortir de sa chambre, comme devant être sans cesse entouré de ses gens? «Jean Jacques Rousseau: Émile ou De l'èducation. Introduction, bibliographie, notes, et index analytique par François et Pierre Richard, Paris 1964, S. 13.

11 Rousseau: Emil (wie Anm. 10), S. 14. Hervorhebung von mir.

12 Rousseau: Émile (wie Anm. 10), S. 12. Die Emphase des Lebens formuliert Rousseau auch vor dem Hintergrund des Todes: "Il s'agit moins de l'empêcher de mourir que de le faire vivre. Vivre, ce n'est pas respirer, c'est agir; c'est faire usage de nos organes, de 
Fähigkeit, sich an sich immer verändernde Umstände anzupassen. Leben heißt, immer gegenwärtig sein zu können. In Rousseaus Überlegungen zur Erziehung künftiger Generationen spielt daher die `Gegenwart ‘ eine zentrale Rolle. Zum einen weil die Erziehung für künftige Gegenwarten vorbereiten soll, zum anderen weil Lernen nach Rousseau nur als Prozess in der Gegenwart, nämlich als konkrete und reale Gegenwartserfahrung möglich ist. Der Zögling soll nicht abstrakt lernen, was die Generationen vor ihm gelernt haben, sondern er soll lernen, jeweils das zu bewältigen, was die jeweilige Gegenwart von ihm verlangt. ${ }^{13}$

Jene Ausgangsbeobachtung, dass die Welt, als Sphäre von Bedingungen, in ständigem Wandel begriffen sei, erscheint auch bei Diderot als Argument für eine Reform. In seiner dramentheoretischen Reformschrift Entretiens sur le fils naturel heißt es: "Bedenken Sie, daß täglich neue Stände entstehen. ${ }^{14}$ »Songez qu'il se forme tous les jours des conditions nouvelles. ${ }^{15}$ Die conditions, die, wie Lessing hier ganz richtig übersetzt, Stände der Gesellschaft, ${ }^{16}$ sind in Bewegung, d.h. in Entwicklung - und deshalb soll das Theater, indem es seine Stoffe nicht aus dem endlichen Reservoir der Charaktere, sondern aus den unendlichen sich verändernder 'Conditions` schöpft, eben diese Veränderungen selbst zum Gegenstand der Darstellung machen - es soll die

nos sens, de nos facultés, de toutes les parties de nous-mêmes, qui nous donnent le sentiment de notre existence. L'homme qui a le plus vécu n'est pas celui qui a compté le plus d'années, mais celui qui a le plus senti la vie. Tel s'est fait enterrer à cent ans, qui mourut dès sa naissance. Il eût gagné d'aller au tombeau dans sa jeunesse, s'il eût vécu du moins jusqu'à ce temps là.« Ebd., S. 13.

13 "Ein Erwachsener muß vieles wissen, dessen Nutzen ein Kind noch nicht einsehen kann. [...] Bemüht euch, das Kind zu lehren, was seinem Alter nützlich ist, und ihr werdet sehen, daß seine Zeit mehr als ausgefüllt ist. [...]. Aber wird dann noch Zeit sein, das zu lernen, was man wissen muß, wenn der Augenblick gekommen ist, sein Wissen auch anzuwenden? Ich weiß es nicht. Was ich aber weiß, ist, daß es unmöglich ist, es früher zu lernen.« Rousseau: Emil (wie Anm. 10), S. 173.

14 Denis Diderot: Unterredungen über den natürlichen Sohn, in: Ders., Lessing: Das Theater des Herrn Diderot, Stuttgart 1986, S. 81-179, hier S. 159.

15 Denis Diderot: Entretiens sur le fils naturel, in: Ders.: Euvres esthétiques. Textes établis, avec introductions, bibliographies, chronologie, notes et relevés de variantes par Paul Vernière, Paris 1968, S. 154.

16 Am Begriff der conditions bzw. der états hängt die gesamte Wirklichkeit des Sozialen, die Geburt, die Rechte und Pflichten, die Ausbildung, die Tätigkeiten/Berufe, die familialen Beziehungen, die Ökonomie, die Reproduktion der Familie und des Staates, das ganze soziale 'Leben', das sich permanent verändert. Zum komplexen Verhältnis von genealogischen und ökonomischen Aspekten bei der Differenzierung der 'Ständeく in der Frühen Neuzeit siehe Heinrich Bosse: Gelehrte und Gebildete - die Kinder des 1. Standes, in: Ders.: Bildungsrevolution 1770-1830, hg. mit einem Gespräch von Nacim Ghanbari, Heidelberg 2012, S. 327-350. 
Zeit der Gegenwart vergegenwärtigen. Der gesamte apparative Aspekt der Theaterreform Diderots, die Gewinnung der Bühne als Raum der Darstellung, das Ensemblespiel und vor allem die sogenannte vierte Wand, ${ }^{17}$ lässt sich so als Effekt einer reflexiven Verzeitlichung verstehen, einer Reflexion auf den Menschen als Produkt seiner zeitlichen, je gegenwärtigen Bedingungen, deren konstitutiver Zusammenhang beobachtet werden soll. ${ }^{18}$ Die Sichtbarmachung dieses Zusammenhangs aus Bedingungen und Formen verfolgt Diderot über die soziale (und familiale) Sphäre hinaus wiederum bis ins Biologische: Jeder lebende Organismus, so sagt es Diderot in seinem Essai sur la peinture, wird durch die Notwendigkeit, sich zu erhalten und sich fortzupflanzen, auf spezifische Weise geformt und verformt - und diese "Difformitäten" - als die Spuren der Tatsache, dass das Leben nie jenseits der es ermöglichenden Bedingungen gedacht werden kann, nie jenseits der Zeit - schreiben sich in seinen Körper ein. ${ }^{19}$ Von dieser biologischen Einsicht in das notwendig bedingende Verhältnis von Lebewesen und Umwelt gelangt Diderot wiederum zu den Ständen bzw. den sozialen Unterschieden, die aufgrund des verschiedenen Alters und der verschiedenen Beanspruchung des Körpers bei der Arbeit auch selbst biologische Unterschiede sind. ${ }^{20}$ Statt nach den akademischen Konventionen zu zeichnen, sollten die Maler die biologisch-soziale Vielfalt der gegenwärtigen Menschen beobachten, »Personen von verschiedenem Alter und Geschlecht, aus allen Ständen [conditions] der Gesellschaft genommen, genug, alle Arten von Naturen. ${ }^{21}$

17 Vgl. Johannes F. Lehmann: Der Blick durch die Wand. Zur Geschichte des Theaterzuschauers und des Visuellen bei Diderot und Lessing, Freiburg i.Br. 2000.

18 Dabei ist der Hausvater, dem Diderot bekanntlich selbst ein Drama gewidmet hat, gerade deshalb ein so interessanter Stand, weil er im Schnittpunkt der sozialen Differenzierung durch Stände selbst steht - und an ihm der soziale Wandel daher besonders sinnfällig wird: "Le père de famille! Quel sujet, dans un siècle tel que le nôtre, où il ne parait pas qu'on ait la moindre idée de ce que c'est qu'un père de famille!« Diderot: Entretiens (wie Anm. 15), S. 154.

19 Vgl. Denis Diderot: Versuch über die Malerei, in: Ders.: Ästhetische Schriften, hg. von Friedrich Bassenge, 2 Bde., Frankfurt a.M. 1968, 1. Bd., S. 635-694, hier S. 636. Diderot spricht von der "geheimen Verbindung « und "notwendigen Verkettungen dieser Difformitäten."

20 »Ich habe niemals gehört, daß man eine Figur übel gezeichnet nenne, wenn sie ihre äußere Organisation deutlich sehen läßt, wenn das Alter, die Gewohnheit und die Leichtigkeit, tägliche Beschäftigungen [fonctions] auszuüben, wohl ausgedrückt ist. Diese Beschäftigungen bestimmen die vollkommene Größe der Figur, die Proportion jedes Gliedes und des Ganzen: daher sehe ich das Kind entspringen, den erwachsenen Mann und den Greis, den wilden sowie den gebildeten Menschen, den Geschäftsmann [magistrat], den Soldaten und den Lastträger.« Ebd., S. 637.

21 Ebd., S. 641. 
Mit dem Blick auf alle Arten von Naturen geht es Diderot - gleichsam als Biologe des Sozialen - darum, die notwendigen Bedingungen der Möglichkeit des Lebens, wie sie gegenwärtig sichtbar sind, mit zum Gegenstand der Kunst zu machen. ${ }^{22}$ Eine solche Kunst, die nicht von der Akademie ausgeht, sondern vom 'Leben', heißt wenig später Kunst des Genies. Das poetische Genie, wie es seit 1770 auf das 'Leben in Opposition zu Rhetorik, sSchrift ihm zugesprochene "Momentaneität«, ${ }^{23}$ nämlich die Fähigkeit, sich in der Gegenwart auf die 'Gegenwart zu beziehen, konturiert. Auf diesen Zusammenhang von Gegenwart, Genie und Leben« komme ich am Ende noch einmal ausführlich zurück.

II.

Das Theater als Medium, das die sozialen Veränderungen, die Stände, d.h. die conditions als die zeitlichen Bedingungen des Lebens darstellen soll und mit ihnen die Zeit der Gegenwart, das ist der Impuls, den Mercier und Herder sowie die Stürmer und Dränger, und hier vor allem Lenz, von Diderot aufgenommen haben. Besonders deutlich formuliert das Louis-Sébastien Mercier in seinem Buch Du Théâtre ou Nouvel essai sur l'art dramatique (1773), zu dessen deutscher Übersetzung von 1776 der junge Goethe einen Anhang aus seiner Brieftasche beigesteuert hat. Mercier schreibt: "Der Mensch, der durch Regierungsformen, Gesetze, Gewohnheiten modificirt wird, wird zum ganz andren Wesen als er erst war. Folglich müßen auch die alten Regeln des Geschmacks sich ändern, und sich den neuen Gewohnheiten, den neuen Begriffen anpaßen laßen. ${ }^{24}$

Der Mensch wird - mit und durch die Zeit - zum ganz anderen Wesen als er war! Auch hier also, in der Sphäre des Sozialen, erscheint jener Gedanke, der die Zeit als Formationskraft reflektiert. Daraus wiederum folgt, dass

22 Vgl. Denis Diderot: Aus dem Salon von 1767, in: Ders.: Ästhetische Schriften (wie Anm. 19), 2. Bd., S. 14-16.

23 Johann Caspar Lavater: Physiognomische Fragmente zur Beförderung der Menschenkenntniß und Menschenliebe, Vierter Versuch, Leipzig/Winterthur 1778, S. 82. Für diesen Hinweis danke ich Peter Schnyder.

24 [Louis Sébastien Mercier]: Neuer Versuch über die Schauspielkunst. Aus dem Französischen [von Heinrich Leopold Wagner]. Mit einem Anhang aus Goethes Brieftasche. Faksimiledruck nach der Ausgabe von 1776, mit einem Nachwort von Peter Pfaff, Heidelberg 1967, S. 199. 
der Schriftsteller Zeitgenosse sein soll und nicht Gelehrter, dass er sich für das Leben seiner Gegenwart interessieren soll. Nur so könne dem Drama ein »Karakter von Nützlichkeit für die Gegenwart « ${ }^{25}$ eingeprägt werden. Mercier fordert in diesem Sinne die Beobachtung und die Darstellung der Gegenwart:

Man hat die Alten studirt, und hat wohl daran gethan; aber bey ihnen wird man keine detallirte Kenntniß der jetzigen Menschen finden. Neue Generationen haben in diese moralische Existenz, in diesen Protheus, der, indem er entschlüpft, alle Gestalten annimmt, große Veränderungen gebracht. ${ }^{26}$

Daraus wiederum folgt der Imperativ, die Darstellung der eigenen Gegenwart an einem erkennbaren Zeitindex auszurichten. Es heißt weiter: »Ich will schlechterdings erkennen können, in welchem Jahr er [der Schriftsteller] sein Werk verfertigt hat." Und Mercier fährt fort: Ich will, dass der Schriftsteller »das Interesse des Augenblicks, in dem er schreibt, nicht aus der Acht lasse; ich will einen Wiederschein von den Geschäfften, die die Nation in Bewegung setzen, bey ihm entdecken; ich will einen Mann hören, der mit dem, was um ihn herum vorgeht, bekannt ${ }^{27}$ ist. Der hier deutlich werdende Zusammenhang zwischen einem zeitlichen Verständnis von Gegenwart und ihrer Darstellung als die bedingende Umwelt der jetzt Lebenden findet sich auch in den Programmatiken von Herder, Lenz und Goethe.

Sowohl biologisch (nach Holbach/Diderot) als auch sozial (nach Rousseau/ Diderot/Mercier) ist der Mensch ein Proteus, der - je nach seinen zeitlichen Bedingungen - ein ganz anderer wird. Im Begriff des Lebens, wie er bei den französischen Materialisten jenseits der präformierten Schöpfungsordnung gedacht wird, als kontingente, selbstbewegliche und evolutionäre Dynamik, und wie er in den Theorien der Epigenese sich in der zweiten Hälfte des 18. Jahrhunderts durchsetzt ${ }^{28}$ spielt die Zeit, und hier insbesondere die Zeit

25 Ebd. Im französischen Original heißt es: „Il reste à imprimer au Drame un caractère d'utilité présente [...]." [Louis Sébastien Mercier:] Du théâtre ou nouvel essai sur l'art dramatique, Amsterdam 1773, S. 149.

26 Mercier: Neuer Versuch (wie Anm. 24), S. 198f. In der Anmerkung zu "moralische Existenz" schreibt Mercier, ebd.: "Die Buchdruckerkunst, das Schießpulver, die Entdeckung der neuen Welt, die Posten, die Wechselbriefe, und das vorgebliche Gleichgewicht Europens haben das ganze alte System über einen Haufen geworfen."

Ebd., S. 200.

28 Vgl. Hubert Thüring: Das neue Leben. Studien zu Literatur und Biopolitik 1750-1938, München 2012. Ebenso Helmut Müller-Sievers: Epigenese. Naturphilosophie im Sprachdenken Wilhelm von Humboldts, Paderborn u.a. 1993, bes. S. 30-54. 
der Gegenwart, eine zentrale Rolle. ${ }^{29}$ An die Stelle der bloßen Wiederholung des Lebens (und der von Gott gesetzten Gattungen) durch die Ausfaltung schon bei der Schöpfung präformierter Keime tritt ein Modell, welches das Leben im Augenblick der Zeugung jeweils neu einsetzen lässt, tritt ein Modell der Bildung, gemäß dem Organismen sich autopoietisch und mittels ihrer \Lebenskraft in der Interaktion mit ihrer Umwelt entwickeln. ${ }^{30} \mathrm{Da}$ das, was in der Interaktion zwischen System und Umwelt passiert, nicht vorhersehbar ist, da es immer andere Umwelten sind, auf die das System in zeitlich immer variierenden Systemzuständen trifft, ist das, was tatsächlich geschieht, gebunden an den jeweiligen Moment der Gegenwart. "Die Epigenese ist wahr, insofern alles Besondere zu seiner Zeit entsteht «, ${ }^{31}$ so formuliert rückblickend Karl Friedrich Burdach. Die »innere Lebenskraft«, so sagt es Herder in metaphorischer Übertragung auf seelische Kräfte, ist selbst gerade definiert als die "Gegenwart des Geistes «. ${ }^{32}$

III.

Diese neue Rolle der Zeit der Gegenwart innerhalb des so gedeuteten Lebensprozesses korrespondiert einer semantischen Verschiebung im Gebrauch des Substantivs 'Gegenwart überhaupt. Dieses hatte nämlich bis ins letzte Drittel des 18. Jahrhunderts noch gar keine zeitliche, sondern allein

${ }^{29}$ Vgl. zur Rolle der Zeit im Begriff des Lebens seit dem ausgehenden 18. Jahrhundert François Jacob: Die Logik des Lebenden. Eine Geschichte der Vererbung, aus dem Französischen von Jutta und Klaus Scherrer, mit einem Nachwort von Hans-Jörg Rheinberger, Frankfurt a.M. 2002 (zuerst Paris 1970), S. 145-194. Vgl. auch Michel Foucault: Die Ordnung der Dinge. Eine Archäologie der Humanwissenschaften, übers. von Ulrich Köppen, Frankfurt a.M. 1994 (zuerst Paris 1966), S. 337: »Aber das Lebendige wird von Anfang an mit den Bedingungen gedacht, die ihm eine Geschichte zu haben gestatten."

30 Die Lebenskraft bzw. den Bildungstrieb hatte vor allem Johann Friedrich Blumenbach in Anschlag gebracht und nach ihm viele andere, siehe hierzu Thüring: Das neue Leben (wie Anm. 28), S. 334-359.

31 Karl Friedrich Burdach: Die Physiologie als Erfahrungswissenschaft, Bd. 1, Leipzig 1826, S. 572 .

32 "Der wachende, gesunde Gebrauch der Sinne, thätiger Verstand in wirklichen Fällen des Lebens, muntere Aufmerksamkeit mit reger Erinnerung, mit schnellem Entschluß, mit glücklicher Wirkung begleitet; sie allein sind das, was wir Gegenwart des Geistes, innere Lebenskraft nennen, die sich also auch mit dem Gefühl einer gegenwärtigen wirksamen Kraft, mit Glückseligkeit und Freude selbst belohnet." Johann Gottfried Herder: Ideen zur Philosophie der Geschichte der Menschheit, in: Ders.: Werke in zehn Bänden, Bd. 6, hg. von Martin Bollacher, Frankfurt a.M. 1989, S. 329. 
räumliche Bedeutung. ${ }^{33}$ Die oben zitierte Forderung Merciers, das Drama solle einen "Karakter der Nützlichkeit für die Gegenwart" gewinnen, ist einer der ersten Belege für einen zeitlichen Gebrauch des Substantivs `Gegenwart<. Bis dahin wird das Wort gebraucht im Sinne von Anwesenheit, Präsenz, im selben Raum sein, mit dem eigenen Körper (bzw. der Seele) auf die präsente Umgebung wirken können: "Der Zustand, da man durch seine eigene Substanz ohne moralische Mittelursachen, ja ohne alle Werkzeuge an einem Orte wirken kann, die Anwesenheit«, so fasst Adelung die Bedeutung von `Gegenwart zusammen. ${ }^{34}$ Bei Zedler ist `Gegenwart ein Begriff, der sich auf die räumliche Position endlicher und nichtnotwendiger Kreaturen bezieht: "Gegenwart; in so ferne sie von Creaturen gesagt wird, bestehet sie in derjenigen Relation, da eine Sache mit der andern so zugleich existiret, daß sie sich mit ihrem Wesen bey derselben entweder nahe oder nicht nahe befindet. ${ }^{35}$ Erst in Wörterbüchern des 19. Jahrhunderts wird die zeitliche Bedeutung dann dominant. ${ }^{36}$

Dass der Begriff `Gegenwart etwa seit 1770 in zeitlicher Verwendung belegt ist, zeigt, dass hier, über den Kontext der Diskurse des Lebens hinaus, offenbar der Bedarf für einen Begriff entsteht, der die eigene bzw. die jetzige Zeit zugleich als die Zeit derjenigen beschreibt, die sie als ihre Gegenwart erleben. Die Bedeutungsverschiebung im Substantiv `Gegenwart ist ein erklärungsbedürftiges Phänomen, das als Symptom für jenen historischen

33 Siehe hierzu schon Niklas Luhmann: Temporalisierung von Komplexität. Zur Semantik neuzeitlicher Zeitbegriffe, in: Ders.: Gesellschaftsstruktur und Semantik. Studien zur Wissenssoziologie der modernen Gesellschaft, Bd. 1, Frankfurt a.M. 1980, S. 235-300, und auf dieser Basis grundlegend, aber bislang eher folgenlos für die Erforschung der Historizität des Begriffs `Gegenwart Ingrid Oesterle: Der Führungswechsel der Zeithorizonte in der deutschen Literatur. Korrespondenzen aus Paris, der Hauptstadt der Menschheitsgeschichte, und die Ausbildung der geschichtlichen Zeit 'Gegenwart‘, in: Studien zur Ästhetik und Literaturgeschichte der Kunstperiode, hg. von Dirk Grathoff, Frankfurt a.M. 1985, S. 11-76. Sowie dies.: "Es ist an der Zeit!« Zur kulturellen Konstruktionsveränderung von Zeit gegen 1800, in: Goethe und das Zeitalter der Romantik, hg. von Walter Hinderer, Alexander von Bormann und Gerhart von Graevenitz, Würzburg 2002, S. 91-121.

34 Johann Christoph Adelung: Grammtisch-kritisches Wörterbuch der hochdeutschen Mundart, Bd. 2, Hildesheim 1970 (= Nachdruck der Ausgabe Leipzig 1796), S. 488.

35 Johann Heinrich Zedler: Grosses vollständiges Universal-Lexicon aller Wissenschaften und Künste, Bd. 10, Graz 1994 (= Nachdruck der Ausgabe Halle 1735), Sp. 594.

36 Johann Christian Lossius: Neues philosophisches allgemeines Real-Lexikon: oder Wörterbuch der gesamten philosophischen Wissenschaften, 2. Bd., Erfurt 1804, S. 215: "Gegenwart. Metaphys. Die unmittelbare Gemeinschaft wird Gegenwart genannt." Zumindest als zweiter Teil der Bedeutung findet sich die zeitliche Bedeutung bei Johann Heinrich Campe: Wörterbuch der Deutschen Sprache, Zweiter Theil, Braunschweig 1808, S. 265 : "Die gegenwärtige Zeit. In der Vergangenheit und Zukunft, wie in der Gegenwart." 
Wandel ernst zu nehmen ist, innerhalb dessen shistorischer Wandek zur Selbstverständlichkeit wird - und zum Begründungsargument für weiteren Wandel. Was genau aber leistet das Substantiv 'Gegenwart gegenüber den älteren adjektivischen Formen wie sheutiger oder jetzige Zeit? Die Gegenwart bezeichnet eine solche gegenwärtige Zeit, die sich nicht nur zufälligerweise von anderen, vergangenen Gegenwarten unterscheidet, sondern die dieses Unterschiedensein im Begriff der Gegenwart immer schon impliziert und mit zum Ausdruck bringt. Mit anderen Worten: Die jetzige Zeit kann, sie muss aber nicht von einer 'früheren Zeit unterschieden sein, die `Gegenwart aber ist immer und notwendig die Gegenwart derer, die sie als ihre Gegenwart erleben - und damit auch immer notwendig verschieden von vergangenen oder zukünftigen Gegenwarten.

Norbert Elias hat die Syntheseleistung dieses seit dem letzten Drittel des 18. Jahrhunderts neuen Zeitbegriffs `Gegenwart (neben den Substantiven >Vergangenheit und `Zukunft) dahingehend bestimmt, dass »die Menschen, auf die sich diese Begriffe beziehen und deren Erfahrung sie zum Ausdruck bringen, ständig im Wandel begriffen sind und daß der Bezug auf Menschen, auf ihre Erfahrung, in die Bedeutung dieser Begriffe eingeht." ${ }^{37}$ Worte wie 'früher` oder sspäter`, die lediglich Differenzen innerhalb einer Chronologie bezeichnen, funktionieren

unabhängig von jeder bestimmten Bezugsgruppe. [...] Der Begriff der Gegenwart dagegen ist die Zeitbestimmung einer lebenden Menschengruppe, die weit genug entwickelt ist, um eine kontinuierliche Geschehensabfolge, gleichgültig ob naturaler, sozialer oder persönlicher Art, auf den Wandel zu beziehen, dem sie selbst unterworfen ist. ${ }^{38}$

Das Substantiv `Gegenwart bringt die Reflexion der subjektiven Erfahrung zeitlicher Veränderung zum Ausdruck, indem es sie zugleich auf eine »übergreifende Vorstellung eines in spezifischem Sinne verstandenen kontinuierlichen Zeitverlaufs « ${ }^{39}$ bezieht. ’Gegenwart meint nicht einfach den Gegensatz der jetzigen Zeit zu einer früheren, der modernen, heutigen Zeit zur Zeit der Antike, sondern `Gegenwart ist ein Reflexionsbegriff für ein temporal sich dauernd mitverschiebendes Set der jeweils jetzigen Bedingungen und der durch sie sich vollziehenden Produktion/Konstitution der jeweils in der Ge-

37 Norbert Elias: Über die Zeit, Frankfurt a.M. 1988, S. 47 (Herv. J.L.).

38 Ebd., S. 49.

39 Elke Uhl: Gebrochene Zeit? Ungleichzeitigkeit als geschichtsphilosophisches Problem, in: Geschichtsphilosophie und Kulturkritik. Historische und systematische Studien, hg. von Johannes Rohbeck und Herta Nagl-Docekal, Darmstadt 2003, S. 50-74, hier S. 54. 
genwart Lebenden, die ihrerseits wissen, dass diese Bedingungen morgen bereits wieder andere sein werden. Wie sich diese Vorstellung einer Zeit der Gegenwart vor dem Hintergrund einer Reflexion auf historischen Wandel noch ohne den Zeitbegriff ,Gegenwart artikuliert, zeigt etwa die folgende geschichtsphilosophische Überlegung aus Adam Weishaupts "Anrede« an die Illuminaten:

Mit jeder dieser Perioden des ganzen Geschlechts lernen die Menschen neue, ihnen vorher unbekannte Bedürfnisse kennen. Jedes neue Bedürfniß ist gleichsam der Saamen, aus welchem eine neue Veränderung, ein neuer Zustand, ein Besserseyn hervor keimt, weil es den Menschen zur Thätigkeit reißt, in ihm den Nisus [die Energie, den Schwung] hervorbringt, solches zu befriedigen, hinwegzuschaffen. Aus jedem befriedigten Bedürfniß entsteht wieder ein neues, und die Geschichte des Menschen Geschlechts ist die Geschichte seiner Bedürfnisse, wie das eine aus dem andern entstanden: und diese Geschichte, diese Abstammung, diese Entwickelung der Bedürfnisse ist die Geschichte der Vervollkommnung des ganzen Geschlechts; denn nach diesen richten sich Kultur, Verfeinerung der Sitten, Entwicklung der schlafenden Geisteskräfte: mit der Entwicklung derselben ändert sich zugleich die Lebensart, der moralische und politische Zustand, die Begriffe von Glückseligkeit, das Betragen der Menschen gegen einander, ihre Verhältnisse unter sich, die ganze Lage der jedesmaligen gleichzeitigen Welt. ${ }^{40}$

Die "ganze Lage der jedesmaligen gleichzeitigen Welt«, das ist der komplexe Ausdruck für genau jenen komplexen Sachverhalt einer Gleichzeitigkeit von jeweils in einer (jedesmaligen) Zeit miteinander verbundener Elemente und Bedingungen einer >Welt $`$, die sich in und durch die Zeit miteinander verändern und deren synchroner Zusammenhang als Abfolge jedesmaliger, d.h. auch voneinander unterschiedener Gleichzeitigkeiten zu denken ist. Mit anderen Worten: als synchrone Schnitte durch einen Zusammenhang von Verhältnissen, zu deren Reflexion der Zeitlichkeit ihrer räumlich metaphorisierten Elemente (Lage, Verhältnisse etc.) der Begriff `Gegenwart`zum Zeitbegriff transformiert wird.

40 Adam Weishaupt: Anrede an die neu aufzunehmenden Illuminatos dirigentes, in: Nachtrag von weitern Originalschriften, welche die Illuminatensekte überhaupt, sonderbar aber den Stifter derselben Adam Weishaupt, gewesen Professor zu Ingolstadt betreffen [...], Zwo Abtheilungen, II, München 1787, S. 44-121, hier S. $53 f$. 
IV.

Wie aber ist diese Verzeitlichung zu erklären, wie kommt es, dass das Substantiv `Gegenwart zeitliche Bedeutung gewinnt, und wie kommt es, dass soziale und nichtsoziale (geographische, physikalische, biologische, physiologische) "Lagen" als je zeitliche Bedingungen der in ihnen lebenden Menschen gedacht werden? Die Denkfigur, die zu erklären ist, besteht aus zwei Komponenten, zum einen aus der Vorstellung, der Mensch sei das Produkt (sozialer und nichtsozialer) Umweltbedingungen, zum anderen aus der Prämisse, diese Umwelt sei selbst zeitlichem Wandel unterworfen. Ein Feld, auf dem diese in sich gedoppelte Denkfigur insbesondere Plausibilität gewinnt, ist das Nachdenken über Prozesse der Bildung, und zwar der Bildung im doppelten Sinne des Wortes: Prozesse der Formation des Lebens (Theorien der Epigenese) sowie Prozesse des Lernens und Lehrens (Schule, Universität, Rede, Schrift und Buchdruck), die nun in enger Analogie zu Prozessen des Lebens gedacht werden. In diesem zweiten Feld entsteht im letzten Drittel des 18. Jahrhunderts ein diskursiver Differenzierungsprozess, der Rede und Schrift nicht mehr als Repräsentationen gleichsetzt, sondern sie scharf $\mathrm{zu}$ unterscheiden beginnt und so ein spezifisches »Problem der Schriftlichkeit ${ }^{41}$ entdeckt. Im Rahmen dieser kritischen Schriftreflexion entsteht eine Opposition zwischen Schrift und ¿Leben`, die für die Plausibilisierung einer Zeit der Gegenwart eine zentrale Rolle spielt.

In seinen Fragmenten über die deutsche Literatur stellt Herder seiner eigenen Zeit die kritische Diagnose des Verfalls durch Schriftlichkeit. In der »alten sinnlichen Zeit der Welt« dagegen,

in jenen rohen Zeiten, wo noch die Seele der Dichter, die zu sprechen, und nicht zu plappern gewohnt war, nicht schrieb, sondern sprach, und auch schreibend lebendige Sprache tönete: in jenen Zeiten, wo die Seele des andern nicht las, sondern hörte, und auch selbst im Lesen, zu sehen und zu hören wußte, weil sie jeder Spur des wahren und natürlichen Ausdrucks offen stand: daher rühren jede Wunder, die die Dichtkunst geleistet, über die wir staunen und fast zweifeln; die aber unsre süße Herren verspotten, und närrisch finden: daher rührt alles Leben der Dichtkunst, was ausstarb, da der Ausdruck nichts als Kunst wurde, da man ihn von dem, was er ausdrücken sollte, abtrennete $\left[\ldots . .{ }^{42}\right.$

41 Heinrich Bosse: Der Autor als abwesender Redner, in: Ders.: Bildungsrevolution (wie Anm. 16), S. 237-249, hier S. 241.

42 Johann Gottfried Herder: Über die neuere deutsche Literatur. Fragmente, als Beilagen zu den Briefen, die neueste Literatur betreffend. Dritte Sammlung. 1767, in: Ders.: Werke in zehn Bänden, hg. von Martin Bollacher u.a., Bd. 1: Frühe Schriften 1764-1772, hg. von 
Herders Opposition von ssprechen versus sschreiben hören ist zugleich eine Opposition, die innerhalb des Umgangs mit Schrift selbst wiederkehrt: In den alten Zeiten tönte schreibend lebendige Sprache, und selbst im Lesen wusste man zu hören und zu sehen. Der »Verfall der Dichterei«, den Herder beklagt, liegt darin begründet, dass "man sie der Mutter Natur entführte, in das Land der Kunst brachte, und als eine Tochter der Künstelei ansah: der Fluch der auf dem Lesen der Alten ruhet, wenn wir bloß Worte lernen [...] ${ }^{4}{ }^{43}$

Es geht also nicht gegen das Lesen von Schrift überhaupt, sondern gegen eine Weise des Lesens, die das Wort nicht als lebendigen Ausdruck einer sich ausdrückenden Seele fasst, sondern als bloßes Zeichen, als rhetorisches Kleid und nicht als beseelten Körper. Herder reflektiert diesen Verlust der Lebendigkeit durch rhetorischen Umgang mit Schrift auch als Erfahrung seines eigenen Lebens. Als er 1769 aus seinem Amt als Rektor in Riga aussteigt und sich auf ein Schiff nach Frankreich begibt, blickt er auf sich selbst und seinen Bildungsgang kritisch zurück. Zwar habe er früh Karriere als Prediger und gelehrter Autor gemacht, gleichwohl wünsche er sich, seine Zeit mit einer anderen Art des Wissens gefüllt zu haben:

Ich beklage mich, ich habe gewisse Jahre von meinem Menschlichen Leben verlohren: und lags nicht blos an mir sie zu genießen? bot mir nicht das Schicksal selbst die ganze fertige Anlage dazu dar? [...] Ich hätte meine Jahre geniessen, gründliche, reelle Wißenschaft kennen, und Alles anwenden gelernt, was ich lernte. Ich wäre nicht ein Tintenfaß von gelehrter Schriftstellerei, nicht ein Wörterbuch von Künsten und Wißenschaften geworden, die ich nicht gesehen habe und nicht verstehe: ich wäre nicht ein Repositorium voll Papiere und Bücher geworden, das nur in die Studierstube gehört. Ich wäre Situationen entgangen, die meinen Geist einschlossen und also auf eine falsche intensive Menschenkänntniß einschränkten, da er Welt, Menschen, Gesellschaften, Frauenzimmer, Vergnügen, lieber extensiv, mit der edlen feurigen Neubegierde eines Jünglinges, der in die Welt eintritt, und rasch und unermüdet von einem zum andern läuft, hätte kennen lernen sollen. Welch ein andres Gebäude einer andern Seele! Zart, reich, Sachenvoll, nicht Wortgelehrt, Munter, lebend, wie ein Jüngling! einst ein glücklicher Mann! einst ein glücklicher Greis! ${ }^{44}$

Ulrich Gaier, Frankfurt a.M. 1985, S. 367-539, hier S. 403. Herder reflektiert in diesem hier zitierten sechsten Abschnitt der dritten Sammlung in historischer und zeichentheoretischer Perspektive das Verhältnis von Gedanke und Ausdruck. Grundlegend hierzu Bosse: Der Autor (wie Anm. 41), S. 237-249.

43 Herder: Fragmente (wie Anm. 42), S. 403.

44 Johann Gottfried Herder: Journal meiner Reise im Jahr 1769, hg. von Katharina Mommsen, Stuttgart 1983, S. $9 f$. 
Die Argumentation stellt hier die gelehrte Schriftstellerei, Wörterbuch, Papiere und Bücher auf der einen der gründlichen und reellen Kenntnis von Welt und Sachen, der Anwendung und dem Genießen auf der anderen Seite gegenüber. Zugleich verknüpft Herder diese Opposition mit der biologischen von Jüngling versus Greis. Der Jüngling lernt nicht Worte auswendig, die er vorfindet und von den Alten übernimmt, sondern er (er)findet, formt und bildet seine eigenen Worte aus dem eigenen Kontakt mit der Welt; zumindest ist das der Wunsch, der bis zur Phantasie der Selbstzerstörung alles nicht selbst Gelernten führt:

o warum ist man durch die Sprache, zu abstrakten Schattenbildern, wie zu Körpern, wie zu existirenden Realitäten verwöhnt? $==$ Wenn werde ich so weit seyn, um alles, was ich gelernt, in mir zu zerstören, und nur selbst zu erfinden, was ich denke und lerne und glaube! $!^{45}$

Und am Ende des Tagebuches heißt es: »Jeder Mensch muß sich eigentlich seine Sprache erfinden, und jeden Begrif in jedem Wort so verstehen, als wenn er ihn erfunden hätte. " ${ }^{46}$ Die Jugend der Seele, das ist für Herder die Fähigkeit, Worte durch Vermittlung der Sinne im gegenwärtigen Moment zu finden: „Und wer seine Muttersprache so lebendig lernte, daß jedes Wort ihm so zur Zeit käme, als er die Sache sieht und den Gedanken hat: welch ein richtiger, philosophisch denkender Kopf! Welch eine junge blühende Seele! ${ }^{47}$

Im Zentrum von Herders Phantasien einer kommenden Vollkommenheit durch Bildung, ${ }^{48}$ im Kern seiner Phantasie einer Realschule ${ }^{49}$ und im Kern seiner Erfindung des Historismus ${ }^{50}$ liegt jenes Verhältnis zwischen aufnehmender und verarbeitender jugendlicher Seele und umgebender Welt als Bildungsfaktor des Lebens - und damit ein Verhältnis, das Herder vom Unterricht durch bloße Abstraktionen und Schriftvermittlung gerade unterscheidet:

Das ist der Weg, Originale zu haben, nehmlich sie in ihrer Jugend viele Dinge und alle für sie empfindbare Dinge ohne Zwang und Präoccupation auf die ihnen eigne Art empfinden zu lassen. Jede Empfindung in der Jugendseele ist nicht blos was sie ist, Materie, sondern auch aufs ganze Leben Materie: sie wird nachher immer ver-

\footnotetext{
45 Ebd., S. 12.

46 Ebd., S. 140.

47 Ebd., S. 140f.

48 Vgl. ebd., S. 20.

49 Vgl. ebd., S. 39-77.

50 Vgl. ebd., S. 30-39.
} 
arbeitet, und also gute Organisation, viele, starke, lebhafte, getreue, eigne Sensationen, auf die dem Menschen eigenste Art[,] sind die Basis zu einer Reihe von vielen starken, lebhaften, getreuen, eignen Gedanken, und d[as] ist das OriginalGenie: Dies ist in allen Zeiten würksam gewesen, wo die Seele mit einer grossen Anzahl starker und eigenthümlicher Sensationen hat beschwängert werden können: in den Zeiten der Erziehung fürs Vaterland, in grossen Republiken, in Revolutionen, in Zeiten der Freiheit, und der Zerrüttungen wars würksam. Diese sind für uns weg: wir sind im Jahrhundert der Erfahrungen, der Polizei, der Politik, der Bequemlichkeit, wo wir wie andre denken müssen, weil wir, was sie sehen, wie sie sehen lernen, und man es uns durch Religion, Politik, Gesellschaftston u.s.w. selbst zu denken verbeut, wie wir wollen. Wir sehen in unsrer Jugend wenige Phänomena, wenn es noch Zeit ist, sie zu sehen, damit sie in uns leben. ${ }^{51}$

Erfunden wird um 1770 nicht nur in Ansätzen ein zeitlicher Begriff von Gegenwart, sondern zugleich der Begriff der Jugend als jene biologisch definierte Lebensphase, deren große physiologische und geistige Energie überhaupt für einen unvermittelten Bezug auf die "gegenwärtige[n] Züge « ${ }^{52}$ der eigenen lokalen Umwelt qualifiziert. Das problematisiert Herder (zunächst) von der biologischen Zeitlichkeit des Subjekts aus: „O Jugend der Seele, die so stark spricht, als sie siehet und fühlet! Mit jeder Wiederholung schwindet ein Zug der Aufmerksamkeit: mit jeder Wiederholung schwächt sich Bild, es wird nur Nachbild, Nachabdruck, und endlich ists die geschwächste Gestalt der Seele. ${ }^{53}$

Die "Jugend der Seele« erscheint als Integral der notwendig nachlassenden Fähigkeit des Lebens, sinnlich Gegenwärtiges als ein solches Gegenwärtiges aufzunehmen und wiederzugeben. Was Herder unermüdlich wiederholt, ist jener unwiederholbare Ursprungsaugenblick der Sprachschöpfung, in dem die (jugendliche) Seele noch keine Sprache hat, aber aufgrund der unmittelbaren sinnlich-besonnenen Bezugnahme auf seine lokale Gegenwart Sprache findet. Es handelt sich um das Modell einer sensualistisch-vitalistischen Semiose: Im Augenblick der intensiv erlebten räumlichen und sinnlichen Gegenwart vollzieht sich - als Prozess des Lebens - die Verarbeitung eines Eindrucks der Außenwelt (der Materie) zu einem individuellen Ausdruck in der Sprache. ${ }^{54}$

51 Ebd., S. $143 f$.

52 Johann Gottfried Herder: Von deutscher Art und Kunst. Einige fliegende Blätter, in: Ders.: Werke (wie Anm. 42), Bd. 2: Schriften zur Ästhetik und Literatur 1767-1781, hg. von Gunter G. Grimm, Frankfurt a.M. 1993, S. 443-549, hier S. 486.

53 Herder: Journal (wie Anm. 44), S. 150. Herder entwickelt die Idee zu einem »Werk über die Jugend und Veraltung Menschlicher Seelen«. Ebd., S. 134.

54 Vgl. hierzu auch Herders Abhandlung über den Ursprung der Sprache (1772). 
Dieser unwiederholbare Augenblick der Zeichenstiftung verdankt sich zunächst einer Emphatisierung der räumlichen Dimension von Gegenwart im Sinne eines Bezugs auf das sinnlich Anwesende. Gerade aber weil der Bezug als Augenblick gedacht wird, der in zeitlicher Opposition zu jeder weiteren Wiederholung steht, kippt die Emphatisierung der räumlichen Dimension der Gegenwart schon hier auch ins Zeitliche. Zugleich wird die Intensität des räumlichen Gegenwartsbezugs an die individuelle Zeit der Kindheit und der Jugend gebunden. Kindheit ist die Zeit, in der die Eindrücke, weil sie neu sind, stark wirken, und Jugendlichkeit ist die Fähigkeit, diese lebendige Stärke auch über den ersten Eindruck und die Vermittlung der Schrift hinaus zu bewahren:

Wem seine ersten Bilder so schwach sind, daß er sie nicht stark und in eben der Stärke von sich geben kann, da er sie empfangen, der ist schwach und alt. So gehts allen Vielbelesnen und Zuviellesenden, die nicht Gelegenheit haben, das was sie gelesen, Einmal stark und lebendig zu wiederholen: oder die nicht Lebhaftigkeit genug haben, zu lesen, als ob man sähe, fühlte, sich selbst empfände, oder anwendete $\left[\ldots . .{ }^{55}\right.$

Man sieht, wie Herder versucht, durch eine Als-ob-Figur jenes 'Leben im Sinne eines unmittelbaren Gegenwartsbezug aus Sehen, Fühlen, Selbstempfinden in die Sphäre des Lesens von Schrift selbst hineinzutragen, die ihm aber "eigentlich" entgegensteht.

Die Jugend, als Manifestation des Lebens und ihrer Kraft schlechthin, muss, so sagt es auch Rousseau, vor dem Medium der Schriftlichkeit geschützt werden: "Kein anderes Buch als die Welt, kein anderer Unterricht als die Tatsachen. Ein Kind, das liest, denkt nicht; es liest nur. Es unterrichtet sich nicht, es lernt nur Worte. ${ }^{56}$ Die Jugend biologisch als Zeitphase spezifischer geistiger Energie zu fassen, die besonders befähigt ist, Gegenwärtiges als Gegenwärtiges wahrzunehmen, das hatte bereits vor Herder Claude-Adrien Helvétius formuliert, indem er seinerseits, in der Folge Rousseaus, geistige Energie an die körperlich fundierten Leidenschaften gebunden hatte, deren Intensität im Alter unaufhaltbar nachlasse. Das sei erkennbar daran, dass "Greise nicht mehr zu jenen kühnen Unternehmen und erstaunlichen Leistungen des Geistes fähig sind, die für die Leidenschaften charakteristisch

55 Herder: Journal (wie Anm. 44), S. 151. Jugend als Begriff, der biologisches Alter auf die geistigen Fähigkeiten projiziert, ist ein Zentralbegriff des gesamten Textes. Siehe auch ebd., S. 134-147.

56 Rousseau: Emil (wie Anm. 10), S. 159. 
sind. $\aleph^{57}$ Die Leidenschaften stellen dabei Energie für Gegenwartsbezüge (in doppeltem Sinne), d.h. für Aufmerksamkeit, bereit: "Die Stärke unserer Aufmerksamkeit entspricht also der Stärke unserer Leidenschaften. ${ }^{58}$ Die Leidenschaften sind es, die uns befähigen, selbst und vom eigenen Interesse getrieben, besonders intensive Beobachtungen und Wahrnehmungen der uns umgebenden Welt zu machen. Der junge Goethe erklärt eben dies zur Voraussetzung der künstlerischen Produktion: »Nur da, wo Vertraulichkeit, Bedürfniß und Innigkeit wohnen, wohnt alle Dichtungskraft, und weh dem Künstler, der seine Hütte verläßt, um in den Akademischen Pranggebäuden sich zu verflattern!« Und weiter: "Was der Künstler nicht geliebt hat, nicht liebt, soll er nicht schildern, kann er nicht schildern. Ihr findet Rubensens Weiber zu fleischig! Ich sage euch, es waren seine Weiber [...] «. ${ }^{59}$

Der Gegenwartsbezug durch die Energie der eigenen Leidenschaft erklärt hier auch, warum Ideale der Kunst nicht zeitlos sind, sondern je aktuell die eigenen Umwelterfahrungen spiegeln. Und so kippt der emphatisierte sinnlich-leidenschaftliche Bezug auf die je gegenwärtige Umwelt ins Zeitliche, wenn die Umwelt ihrerseits durch und in der Zeit eine andere wird. Mercier schreibt:

Nur die Pedanten, [...] nur Schulfüchse können ausrufen, es leben die Griechen! es leben die Griechen! Der verständige Mann wird sagen: SSeht eure Landsleute, oder schreibt nicht; ihr sollt nicht den Menschen überhaupt, den Menschen aus dem Zeitalter, aus dem Land sollt ihr schildern. Wer nicht damit anfangen wird die Menschen, mit welchen er lebt, kennen zu lernen, wird in der theatralischen Kunst keine großen Sprünge machen. ${ }^{60}$

Gegenwart wird, zeitlich verstanden, zu einer Metonymie des alten Raumbegriffs, einfach weil er nun die Anwesenheit unter dem Aspekt der zeitlichen Veränderung fasst. ${ }^{61}$

57 Claude-Adrien Helvétius: Vom Geist, übers. von Theodor Lücke, Berlin/Weimar 1973, S. 393.

58 Ebd., S. 281.

59 Johann Wolfgang Goethe: Nach Falkonet und über Falkonet, in: Mercier: Neuer Versuch (wie Anm. 24), S. 487-496, hier S. 494.

60 Mercier: Neuer Versuch (wie Anm. 24), S. 199.

61 Diese Zeit wird dann in neuer Weise auch wieder an den Raum gebunden, sei es an die jeweilige Region oder aber an die Nation bzw. die Welt. Gegenwart als Zeit, die wie ein synchroner Schnitt durch alle sozialen Verhältnisse funktioniert, impliziert auch, dass diese sozialen Verhältnisse einen ständeübergreifenden (lokalen, regionalen, nationalen oder globalen) Zusammenhang bilden und hier alle betreffen. Gerade dieser Punkt wird dann zur allgemeinen Erfahrung durch die Französische Revolution. Man kann nicht mehr nicht betroffen sein. 
Im Diskurs über das Genie wird jene sensualistisch-vitalistische Semiose, jene Bildung des Genies durch das 'Leben und jenseits gelehrten Unterrichts durch Worte und Wissenschaften immer wieder auch auf die spezifischen Lebenserfahrungen des Genies zurückgeführt. Die Vorstellung, dass sich das Genie bzw. der wahre Dichter - jenseits einer Ausbildung durch das Medium Schrift ${ }^{62}$ - von früh an auf seine gegenwärtige Umwelt beziehen und diese im Medium der Schrift dann als seine Welt darstellen soll, prägt den Diskurs des poetischen Genies um $1770 .^{63}$ Zum ersten Aspekt: Das Genie bezieht seinen Stoff aus der Gegenwart, seine inventio orientiert sich nicht an überlieferten Topoi, sondern an der sinnlichen Gegenwart, die es selbst gleichsam kreativ verstoffwechselt. Unter der Frage »Was soll der Dichter studieren?" polemisiert daher auch Mercier gegen die rhetorische Ausbildung des Schriftstellers. Der Dichter soll seine Bücher beiseitelegen und die Welt, die ihn umgibt, seine Gegenwart, intensiv beobachten: "Was kann mir interessanter seyn als dasjenige, was in dem Augenblick vorgeht, in dem ich schreibe! ${ }^{64}$

Das Schreiben beginnt nicht mehr beim Lesen der Schriften (der Alten), sondern beim Leben: An die Stelle des "gelehrten Schulfuchses" tritt das Genie als Autodidakt: Diese Theorie des Genies als Autodidakt formuliert in aller Deutlichkeit bereits Christoph Martin Wieland in seiner biographi-

62 Wenn es immer wieder heißt, Genie sei nicht lernbar, sondern angeboren (vgl. Lavater: Physiognomische Fragmente [wie Anm. 23], S. 80), dann arbeitet hier genau jene Opposition von Schrift und Leben. Das Genie kann Genie nicht durch Schrift werden. Die Kategorie des Angeborenen heißt aber nicht, dass sich die Fähigkeiten des Genies nicht entwickeln müssten, hier wird Anlage und Entwicklung noch nicht getrennt. Das Genie lernt, indem es lebt, mit der Elastizität seiner Seele, seiner Sinne und seines Nervensystems, immer gegenwärtig zu sein (vgl. ebd., S. 81). Vgl. zur fehlenden Unterscheidung von Anlage und Entwicklung im 17. und 18. Jahrhundert: Hans-Jörg Rheinberger/Staffan Müller-Wille: Vererbung. Geschichte und Kultur eines biologischen Konzepts, Frankfurt a.M. 2009, S. 39.

63 Das steht im Gegensatz zu noch kurz zuvor formulierten Versuchen, das Genie innerhalb der Schönen Wissenschaften zu verorten: »Allein, was vermag das Genie ohne Unterricht, ohne Kunst, ohne Uebung? Was wird der größte Geist treffliches hervorbringen, wenn er noch nicht durch Wissenschaften gebildet, noch nicht mit einem Vorrathe schöner und nützlicher Gedanken ausgerüstet, mit einer Menge lebhafter Bilder ausgeschmückt.« Christian Fürchtegott Gellert: Von dem Einflusse der schönen Wissenschaften auf das Herz und die Sitten. Eine Rede bey dem Antritte der Profession. Übers. aus dem Lateinischen, in: Ders.: Sämtliche Schriften, Siebender Theil, Reuttlingen 1775, S. 76-93, hier S. 79.

64 Mercier: Neuer Versuch (wie Anm. 24), S. 235. 
schen Skizze zu Shakespeare, die er 1766 seinen Übersetzungen von dessen theatralischen Werken beigibt. ${ }^{65}$ Gerade das Fehlen einer "claßischen Erziehung" ist demnach der Grund dafür, dass Shakespeare ein Genie wurde: »Es ist mehr als eine blosse Vermuthung, daß es diesem kleinen Umstand, dem Mangel einer claßischen Erziehung, zuzuschreiben ist, daß wir einen Shakespear haben. ${ }^{66}$ Zwar hätte eine klassische Ausbildung, so Wieland, "seinen Geist gebildet [...], aber mit allen diesen Vortheilen würde er nicht mehr Shakespear gewesen seyn; nicht mehr der ursprüngliche Genie [...]. «77 Und nun setzt Wieland gegen die Schulausbildung durch Texte eine Lebenssausbildung durch unmittelbare, nämlich nicht durch Schrift vermittelte Erfahrung:

[E]igne Betrachtungen; scharfe Sinnen, als die Werkzeuge dazu; eine genaue Aufmerksamkeit auf die unmittelbaren Eindrüke, welche die Gegenstände auf ihn machen - das ist es, was den Genie entwikelt, was seine Nerven spannt und belebt, was ihm durch die reineste Nahrung, die er unmittelbar gleichsam aus der Brust der Natur zieht, zu diesem edeln Wuchs und zu dieser gesunden Fülle bringt, welche die wahren Genien so stark von den Nachahmern (selbst von denen, welche würklich Genie haben) unterscheiden. ${ }^{68}$

Am weitesten ging in dieser Provokation der alteuropäischen Vorstellung, dass man Autorschaft in der Schule im Medium der Schrift lerne, sicher Edward Young in seinen Gedanken über das Original-Genie, wenn er behauptet, dass es vielleicht sogar "manches Genie gegeben, welches weder lesen noch schreiben konnte ${ }^{69}$

In dem Maße, wie man über die jeweiligen Bedingungen nachdenkt, unter denen das Genie am ehesten erweckt werden kann, entsteht nun, das

65 Wieland gibt hier weitgehend eine Übersetzung der Biographie von Rowe, fügt aber gerade jene Gedanken über das Genie, um die es im Folgenden geht, selbständig hinzu. Christoph Martin Wieland: Einige Nachrichten von den Lebens-Umständen des Herrn Willhelm Shakespear, in: Ders.: Gesammelte Schriften, 2. Abt.: Übersetzungen II (3): Shakespeares theatralische Werke. Sechster bis achter Teil, hg. von Ernst Stadler, Hildesheim 1987 (= Nachdruck der Ausgabe Berlin 1911), S. 558-569.

66 Ebd., S. 560.

67 Ebd. Dieses Argument zur Biographie Shakespeares findet sich auch bei Alexander Gerard: Versuch über das Genie. Übers. aus dem Englischen von Christian Garve, Leipzig 1776, S. 16f. Für Mercier sind die Gelehrten Gesellschaften allenfalls gut, »eine Nation der Unwissenheit zu entreißen, und sie zu Mittelmäßigen zu bringen; aber hernach ersticken sie auch alles Genie [...] Ein Genie hat jederzeit die Schulen hinter sich gelaßen, oder hat sie gar verachtet." Mercier: Neuer Versuch (wie Anm. 24), S. 402.

68 Wieland: Einige Nachrichten (wie Anm. 65), S. 561.

${ }^{69}$ Edward Young: Gedanken über die Original-Werke, aus dem Englischen übers., Heidelberg 1977 (= Nachdruck der Ausgabe Leipzig 1760), S. 35. 
betrifft den zweiten Aspekt, die Idee, die Aufgabe des durch solche Bedingungen entstandenen Genies sei wiederum, die Welt als gegenwärtiges Bedingungsgefüge abzubilden, d.h. die jedesmalige gleichzeitige Lage der Welt darzustellen, wie sie ist, d.h., sie zu dokumentieren. Das formuliert deutlich Jakob Michael Reinhold Lenz: »Der wahre Dichter verbindet nicht in seiner Einbildungskraft, wie es ihm gefällt, was die Herren die schöne Natur zu nennen belieben, was aber mit ihrer Erlaubnis nichts als die verfehlte Natur ist. Er nimmt Standpunkt - und dann mufs er so verbinden. $^{70}$

Vor dem Hintergrund der üblichen Nacherzählungen des Geniediskurses ist das eine irritierende Äußerung, denn es handelt sich um eine Unfreiheitserklärung. Die Rede ist in ihr nicht von der autonomen Phantasie und der absoluten Freiheit des Genies, das sich von allen Regeln abkoppelt, sondern Lenz ersetzt eine Regelquelle durch eine andere. An die Stelle der in Büchern überlieferten ästhetischen Beschränkungsregeln für die künstlerische Produktion tritt die empirisch erfahrbare gegenwärtige Welt als Beschränkungsregel. Der wahre Dichter ist frei, den Standpunkt zu wählen, von dem er die ihm zeitgenössische Welt und die Bedingungsketten in ihr anschaut. Einmal gewählt, ist aber das, was er mit seiner besonderen Auffassungs- und Zusammenfassungsfähigkeit sieht, die Beschränkungsregel seiner Wiedergabe, dann muss er so verbinden!

Der Sinn dieser neuen Beschränkungsregel liegt offenbar darin, den Blick und die besondere Wahrnehmungsfähigkeit des Genies auf die Gegenwart auszurichten. Auch hier also ist nicht die Schrift und die Überlieferung der Vergangenheit Quelle des Schreibens, sondern die gegenwärtige Welt, mit ihren komplexen Relationen und Bedingungsverhältnissen, die als gegenwärtige dokumentiert werden soll. Nach Kant, der >Welt‘ als Totalität von Bedingungen fasst, ist diese für die Wahrnehmung ein zeitliches Problem, denn jene Bedingungstotalität kann eigentlich nur im zeitlichen Nacheinander überhaupt nachvollzogen werden. ${ }^{71}$ Das Genie aber kann jede Art von Komplexität mit besonderer Schnelligkeit in ein Bild zusammenfassen. Bei Lenz lautet der entsprechende Gedanke:

Wir nennen die Köpfe Genies, die alles, was ihnen vorkommt, gleich so durchdringen, durch und durch sehen, daß ihre Erkenntnis denselben Wert, Umfang, Klarheit hat, als ob sie durch Anschaun oder alle sieben Sinne zusammen wäre er-

70 Jakob Michael Reinhold Lenz: Anmerkungen übers Theater, in: Ders.: Werke und Briefe, hg. von Sigrid Damm, Bd. 2, Frankfurt a.M. 1992, S. 648.

71 Vgl. hierzu Thomas Khurana: Idee der Welt. Zum Verhältnis von Welt und Bild bei Kant, in: Soziale Systeme 18 (2012), H. 1/2, S. 94-118. 
worben worden. Legt einem solchen eine Sprache, mathematische Demonstration, verdrehten Charakter, was ihr wollt, eh ihr ausgeredt habt, sitzt das Bild in seiner Seele, mit allen seinen Verhältnissen, Licht, Schatten, Kolorit dazu. ${ }^{72}$

Bei Lavater: "Nenn's ungewöhnliche Schnelligkeit des Geistes, entfernte Verhältnisse mit glücklicher Ueberspringung der Mittelverhältnisse zusammenzufassen. ${ }^{73}$ Für diese Erfassung der Welt als eines dynamischen und produktiven Lebensbedingungszusammenhanges muss das Genie eine spezifische, nämlich nur durch sich selbst vermittelte Lebenskraft haben, "aller Genieen Wesen und Natur ist [...] Selbstleben! " ${ }^{74}$ Dies wiederum ist physiologisch fundiert und auf den Begriff des Lebens bezogen:

Nenn's Elastizität der Seele, oder der Sinne und des Nervensystems - die leicht Eindrücke annimmt, und mit einem schnell ingerirten Zusatze lebendiger Individualität zurückschnellt. [...] Nenn's lebendigen und lebendig machenden Geist, der sein Leben fühlt, und leicht und vollkräftig mittheilt; sich in alles hineinwirft mit Lebensfülle, mit Blitzeskraft. ${ }^{75}$

Für Lavater ist diese Art der lebendigen Wahrnehmung und Darstellung des Lebens selbst eine Zeitfigur, insofern das Genie die Gegenwart als Medium des Zukünftigen erkennt: "Nenn's Ahndung des Unsichtbaren im Sichtbaren, des Zukünftigen im Gegenwärtigen." ${ }^{76}$

VI.

Ich fasse zusammen: Auf zwei Feldern wird Mitte des 18. Jahrhunderts formuliert, dass die Bedingungen des Lebens sich in der Zeit wandeln, sowohl auf dem Feld der biologischen als auch auf dem der sozialen Bedingungen. Die biologischen und die sozialen Formen des Lebens erscheinen so als das Produkt dieser Veränderungen in und durch die Zeit. Der Begriff des Le-

72 Lenz: Anmerkungen (wie Anm. 70), S. 648. Ganz ähnlich bei Holbach: System der Natur (wie Anm. 4), S. 111: "Als Geist bezeichnen wir in der Tat die Fähigkeit, mit der einige Wesen unserer Gattung die Gesamtheit der Gegenstände und ihre verschiedenen Beziehungen leicht und schnell auffassen. Als Genie bezeichnen wir die Fähigkeit, diese Gesamtheit und diese Beziehungen in ausgedehnten, nützlichen und schwer erkennbaren Gegenständen leicht zu überblicken."

73 Lavater: Physiognomische Fragmente (wie Anm. 23), S. 81.

74 Ebd., S. 83.

75 Ebd., S. 81 .

76 Ebd. 
bens, wie er sich in der zweiten Hälfte des 18. Jahrhundert als epigenetischer Begriff etabliert, beschreibt wesentlich einen Prozess, in dem lebendige Organismen (mit notwendig nachlassender Kraft) in der jeweils gegenwärtigen Interaktion mit ihrer Umwelt sich selbst bilden. Der Aspekt der >Gegenwart in dieser Interaktion wird in Diskursen der Bildung zugleich in Opposition zur Vermittlung durch Schrift gesetzt und auf die biologische Eigenzeit der Individuen (Jugend versus Alter) bezogen. Der intensive Bezug der jugendlichen sSeeler auf ihre Außenwelt (als das räumlich Gegenwärtige) geht durch Wiederholung und Vermittlung durch Zeichen, die schon da sind, verloren. In dem Maße, in dem `Gegenwart (im Sinn der sinnlichen Präsenz von Erfahrungsgegenständen) als Bedingung von Bildungsprozessen gedacht wird, wird das Gegenwärtige zur `Gegenwart verzeitlicht: und zwar zum einen auf der Seite des Subjekts und seiner Eigenzeit der biologisch nachlassenden Kraft, die `Gegenwart « erfassen zu können, und zum anderen auf der Seite des Zusammenhangs von Subjekt und seiner jeweiligen, sich in der Zeit verändernden Welt bzw. Umwelt. Das Genie ist durch Kontakt mit dem, was seine Gegenwart konstituiert, Genie geworden und daher seinerseits befähigt, ’Gegenwart< darzustellen. Diese Gegenwart ist - als Summe der sich jeweils verändernden Lage - die Zeit der Gegenwart. 
